\title{
Research on Hyperbola Fitting Algorithm for Turbulence Level Measurement Test Data
}

\author{
Yufeng Du $\mathbb{D}^{1},{ }^{1}$ Long Wu, ${ }^{2}$ Xunnian Wang, ${ }^{3}$ Jun Lin, ${ }^{1}$ and Neng Xiong ${ }^{1}$ \\ ${ }^{1}$ High Speed Aerodynamics Institute, China Aerodynamics Research and Development Center, Mianyang 621000, China \\ ${ }^{2}$ Science and Technology on Scramjet Laboratory, China Aerodynamics Research and Development Center, \\ Mianyang 621000, China \\ ${ }^{3}$ State Key Laboratory of Aerodynamics, China Aerodynamics Research and Development Center, Mianyang 621000, China \\ Correspondence should be addressed to Yufeng Du; 1415776643@qq.com
}

Received 24 April 2020; Revised 19 June 2020; Accepted 1 July 2020; Published 23 October 2020

Academic Editor: Luis J. Yebra

Copyright (C) 2020 Yufeng Du et al. This is an open access article distributed under the Creative Commons Attribution License, which permits unrestricted use, distribution, and reproduction in any medium, provided the original work is properly cited.

\begin{abstract}
Hyperbola fitting of test data is an extremely important process in turbulence level measurement test in wind tunnels. The solution of the overdetermined equations (SOE) method is often used to solve hyperbola fitting parameters to obtain turbulence level. However, due to unsteady flow characteristics, the SOE method often results in overfitting phenomena, which makes it impossible to solve turbulence level accurately. This paper proposes using the constrained least-squares (CLS) method to convert the problem of hyperbola fitting of test data into the inequality constrained optimization problem and then using the Lagrange programming neural network (LPNN) method to solve turbulence level iteratively. The stability of the LPNN method is analysed, and three sets of typical turbulence level measurement test data are processed using the LPNN method. The results verify the feasibility of applying the LPNN method to iteratively solve the turbulence level of wind tunnels.
\end{abstract}

\section{Introduction}

Wind tunnel test is the most effective method for aerodynamic research. Even if the computer-based numerical simulation technology and the model flight test technology are rapidly improving, wind tunnel test is still an indispensable method for the research of complex aerodynamic characteristics during the research and development of aircraft. The precise design of advanced aircraft requires high accuracy of wind tunnel test results. However, there exist many factors and phenomena which finally result in the inaccuracy of measurements in the wind tunnel, such as the influence of flow qualities, Reynolds and Mach numbers, standard model test results, wind tunnel walls, and supporting system interference in $2 \mathrm{D}$ and $3 \mathrm{D}$ tests $[1-7]$.

As an important flow quality in wind tunnels, turbulence level will affect the accuracy of wind tunnel test results, such as calculation of aircraft force and moment coefficients [1], measurement of test model attitude angle [8], and measurement of transition characteristics of the boundary layer on test model surface [9]. For aircraft design, the errors in wind tunnel tests mean that there will be design errors in aerodynamic parameters such as lift and drag coefficients. The design errors will lead to estimation errors in aircraft weight, which severely restricts the economy and safety of aircraft [10]. Therefore, it is very important to evaluate the turbulence level in wind tunnels accurately and quantitatively.

Hot-wire anemometry (HWA) is currently the most widely used method for turbulence level measurement due to its advantages such as high-frequency response, high sensitivity, and cost effectiveness [11-14]. In the compressible flow, the hot-wire response function derived by the changing overheat ratio method conforms to a hyperbolic relationship. Therefore, the problem of solving turbulence level can be converted into the problem of solving hyperbola fitting parameters of a set of two-dimensional scattered points [15]. However, in the actual measurement and data acquisition process, the two-dimensional scattered points may deviate from hyperbolic distribution due to unsteady 
flow characteristics. In some cases, there are even straight lines and other conic distributions, which make it difficult to solve turbulence level precisely.

Many researchers have studied hyperbola fitting, and the methods utilized differ due to different application scenarios. Hough transform (HF) is a method of graph detection and fitting based on pattern recognition. The HF method converts scattered points in the image space to those in the parameter space and converts the problem of scattered points fitting to the problem of solving extreme value in the parameter space, thereby realizing the detection and fitting of the hyperbola. The HF method has the advantages of strong robustness, but its shortcomings of low computational efficiency limit its real-time application [16-20]. The least-squares (LS) method is the most commonly used method for hyperbola fitting. The key idea of the LS method is to establish a suberror function for each scattered point and use the sum of the squares of all suberror functions as the error function of hyperbola fitting. The suberror function can be expressed by residuals or orthogonal distances between scattered points and fitted hyperbolic curves. Hyperbolic curves are obtained by calculating the extreme value of the error function. The LS method has been widely used due to its ease of application and high computational efficiency [21-25]. Both Lebiga VA $[12,26,27]$ and Radespiel $R[28-31]$ have done a lot of research in the field of turbulence level measurement and obtaining the fitting solution in compressible flow. They conducted a comprehensive and thorough analysis of fluctuation measurement results, which provided data support for the uncertainty analysis of subsequent wind tunnel tests. However, they have not conducted too much research on the hyperbola fitting method. Instead, the solution of the overdetermined equations (SOE) method is used directly to calculate the hyperbolic parameters to complete the hyperbola fitting. The SOE method is based on the LS method, which means it has high computational efficiency, while the SOE method cannot obtain precise turbulence level results due to overfitting if the scattered points deviate from hyperbolic distribution.

In order to solve the problem mentioned above, this paper proposes using the constrained least-squares (CLS) method to convert the problem of hyperbola fitting to the inequality constrained optimization problem and then using the Lagrange programming neural network (LPNN) method to solve turbulence level iteratively. The results show that the LPNN method is superior to the traditional SOE method, which verify the feasibility of the LPNN method for solving turbulence level in wind tunnels.

\section{The SOE Method}

According to the literature [26-32], the response function of the constant temperature hot-wire anemometer (CTA) in compressible flow is

$$
\frac{\Delta E}{\bar{E}}=F_{\mathrm{CTA}} \frac{\Delta m}{\bar{m}}-G_{\mathrm{CTA}} \frac{\Delta T_{0}}{T_{0}},
$$

where $E$ is the output voltage of CTA, $m$ and $T_{0}$ are the gas mass flow rate and total temperature of the hot-wire probe measuring point, and $F_{\mathrm{CTA}}$ and $G_{\mathrm{CTA}}$ are the mass flow rate and total temperature sensitivity coefficients of CTA, respectively. $F_{\mathrm{CTA}}$ and $G_{\mathrm{CTA}}$ are only related to the overheating ratio, namely, the working temperature, of the hot-wire probe under a fixed flow condition. Dividing equation (1) by $G_{\text {СтA }}$ and defining $\theta=1 / G_{\text {СТА }} \Delta E / \bar{E}$ as the response function and $r=F_{\mathrm{CTA}} / G_{\mathrm{CTA}}$ as the independent variable, we can get

$$
\theta=\frac{\Delta m}{\bar{m}} r-\frac{\Delta T_{0}}{\overline{T_{0}}} \text {. }
$$

Take the mean square value of equation (2):

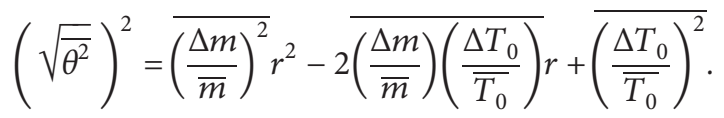

From equation (3), we can know that the response function of CTA conforms to the hyperbolic relationship with $r$ as the independent variable and $\sqrt{\theta^{2}}$ as the dependent variable. The two-dimensional scattered points $(\mathbf{R}, \Theta)$ to be fitted can be obtained by continuously changing the overheat ratio $n$ times and recording the output voltage of CTA at the same time:

$$
\begin{aligned}
& \mathbf{R}=\left[\begin{array}{llll}
r_{1} & r_{2} & \ldots & r_{n}
\end{array}\right]^{T}, \\
& \boldsymbol{\Theta}=\left[\begin{array}{llll}
\sqrt{\sqrt{\theta_{1}^{2}}} & \sqrt{\theta_{2}^{2}} & \ldots & \sqrt{\theta_{n}^{2}}
\end{array}\right]^{T} .
\end{aligned}
$$

Construct the coefficient matrix $\mathbf{A}$, the variable vector $\mathbf{X}$ to be sought, and the nonhomogeneous term vector $\Pi$ of overdetermined equations:

$$
\begin{aligned}
& \mathbf{A}=\left[\begin{array}{ccc}
r_{1}^{2} & r_{1} & 1 \\
r_{2}^{2} & r_{2} & 1 \\
\vdots & \vdots & \vdots \\
r_{n}^{2} & r_{n} & 1
\end{array}\right],
\end{aligned}
$$

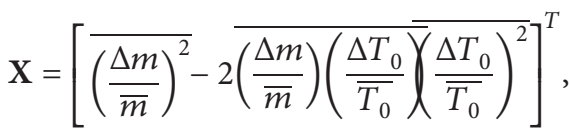

$$
\begin{aligned}
& \boldsymbol{\Pi}=\left[\left(\sqrt{\overline{\theta_{1}^{2}}}\right)^{2}\left(\sqrt{\overline{\theta_{2}^{2}}}\right)^{2} \ldots\left(\sqrt{\overline{\theta_{n}^{2}}}\right)^{2}\right]^{T} .
\end{aligned}
$$

Therefore, the flow fluctuations can be obtained by solving the following overdetermined equations:

$$
\mathbf{A} \cdot \mathbf{X}=\boldsymbol{\Pi} \text {. }
$$

Since $\mathbf{A}$ is not a square matrix, it is impossible to solve the overdetermined equations by solving the inverse matrix of A. Instead, the generalized inverse matrix of A can be solved by the singular value decomposition (SVD) method to solve the overdetermined equations. Any real matrix $\mathbf{A}$ $(n \times q)$ can be decomposed: 


$$
\mathbf{A}=\mathbf{U} \cdot\left[\begin{array}{cc}
\mathbf{W}_{\mathbf{z}} & 0 \\
0 & 0
\end{array}\right] \cdot \mathbf{V}^{T},
$$

where $\mathbf{U}(n \times n)$ and $\mathbf{V}(q \times q)$ are orthogonal matrices, $\mathbf{W}_{z}=\left[\operatorname{diag}\left(w_{1}, w_{2}, \ldots, w_{z}\right)\right], w_{1}, w_{2}, \ldots, w_{z}$ are the nonzero singular values of matrix $\mathbf{A}$, and $w_{1} \geq w_{2} \geq \cdots \geq w_{z}>0$, $z$ is the rank of $\mathbf{A}$. When the rank of $\mathbf{A}$ is full, that is, $z=q$, the SVD form of $\mathbf{A}$ is

$$
\mathbf{A}=\mathbf{U} \cdot\left[\begin{array}{c}
\mathbf{W}_{\mathbf{z}} \\
0
\end{array}\right] \cdot \mathbf{V}^{T} .
$$

According to equation (8), the generalized left inverse matrix of $\mathbf{A}$ is

$$
\mathbf{A}^{+}=\mathbf{V} \cdot\left[\begin{array}{ll}
\mathbf{W}_{\mathbf{z}}^{-1} & 0
\end{array}\right] \cdot \mathbf{U}^{T},
$$

which satisfies $\mathbf{A}^{+} \cdot \mathbf{A}=\mathbf{I}$. Therefore, the solution of the overdetermined equations (6) using the SVD method is

$$
\mathbf{X}=\mathbf{A}^{+} \cdot \Pi \text {. }
$$

After obtaining the flow fluctuations, the following equations can be used to solve the turbulence level Tu of the flow:

$$
\begin{aligned}
\frac{\Delta m}{\bar{m}} & =\frac{\Delta(\rho u)}{\overline{\rho u}}=\frac{\Delta \rho}{\bar{\rho}}+\frac{\Delta u}{\bar{u}}, \\
\frac{\Delta T_{0}}{\overline{T_{0}}} & =\alpha \frac{\Delta T}{\bar{T}}+\beta \frac{\Delta u}{\bar{u}}, \\
\alpha & =\frac{1}{1+(\gamma-1 / 2) M^{2}} \\
\beta & =\frac{(\gamma-1) M^{2}}{1+(\gamma-1 / 2) M^{2}}, \\
\frac{\Delta p}{\bar{p}} & =\frac{\Delta \rho}{\bar{\rho}}+\frac{\Delta T}{\bar{T}}, \\
\frac{\Delta \rho}{\bar{\rho}} & =\frac{1}{\gamma} \frac{\Delta p}{\bar{p}},
\end{aligned}
$$

where $\rho, u, T$, and $p$ are the gas density, velocity, static temperature, and static pressure of the hot-wire probe measuring point, respectively, and $M$ is the Mach number. Turbulence level $T u$ can be solved by the following equations:

$$
\begin{aligned}
& T u=\left(\overline{\left(\frac{\Delta u}{\bar{u}}\right)^{2}}\right)^{1 / 2}=\left(\left[\begin{array}{lll}
H^{2} & H G & G^{2}
\end{array}\right] \cdot \mathbf{X}\right)^{1 / 2}, \\
& H=\frac{1}{1-M^{2}}, \\
& G=\frac{M^{2}}{1-M^{2}} \cdot \frac{1}{\beta} .
\end{aligned}
$$

In the actual measurement process, conventional wind tunnels cannot guarantee the strict steadiness of flow due to the long duration of the turbulence level measurement test by using the changing overheat ratio method. Therefore, there will be cases where the two-dimensional scattered points do not strictly obey hyperbolic distribution, and even the distribution is similar to straight lines and other conic curves. The schematic diagram is shown in Figure 1.

The $O$ points and the solid blue line in Figure 1 are the scattered points under ideal conditions and the hyperbola obtained by the SOE method, and the + points and the dotted red line are the scattered points with flow disturbances and the curve obtained by the SOE method. As a result of overfitting, the fitting curve is approximately a straight line, which has deviated from the ideal hyperbola. In this situation, the SOE method will not accurately solve the turbulence level.

\section{Proposed Algorithm for Solving Turbulence Level}

In order to solve the problem mentioned above, the CLS method is used to convert the problem of hyperbola fitting to the inequality constrained optimization problem, and then the LPNN method is used to obtain turbulence level iteratively.

3.1. The CLS Method. Compared with the traditional LS method, the CLS method can limit the parameters to be fitted within a certain range to prevent overfitting. Suppose that the fitted hyperbolic equation of the two-dimensional scattered points $(\mathbf{R}, \Theta)$ is

$$
f^{2}(r)=c_{1} r^{2}+c_{2} r+c_{3},
$$

where $r$ is the independent variable, $f(r)$ is the dependent variable, and $c_{1}, c_{2}$, and $c_{3}$ are the hyperbola fitting coefficients. According to equation (14), the dependent variable fitting values $\mathbf{F}(\mathbf{R})$ and residuals $\mathbf{E}$ are

$$
\begin{aligned}
\mathbf{F}(\mathbf{R}) & =\left[f\left(r_{1}\right), f\left(r_{2}\right), \ldots, f\left(r_{n}\right)\right]^{T}, \\
\mathbf{E} & =\boldsymbol{\Theta}-\mathbf{F}(\mathbf{R})=\left[e_{1}, e_{2}, \ldots, e_{n}\right]^{T}, \\
e_{i} & =\sqrt{\theta_{i}^{2}}-f\left(r_{i}\right), \quad(i=1,2, \ldots, n) .
\end{aligned}
$$

Select the error function $S$ of the CLS method as the sum of squares of the residuals of each scatter:

$$
S=\sum_{i=1}^{n} e_{i}^{2}
$$

Comparing equations (3) and (14), it can be seen that the hyperbola fitting coefficients $c_{1}, c_{2}$, and $c_{3}$ in the CLS method are the flow fluctuations to be sought; namely,

$$
\mathbf{C}=\left[c_{1}, c_{2}, c_{3}\right]^{T}=\widehat{\mathbf{X}} .
$$

According to the physical meanings of the variables in equations (5) and (17), the constraints are established as follows:

(1) Since the mean square values of $m$ and $T_{0}$ fluctuations are always positive, we can say $c_{1}>0$ and $c_{3}>0$. 


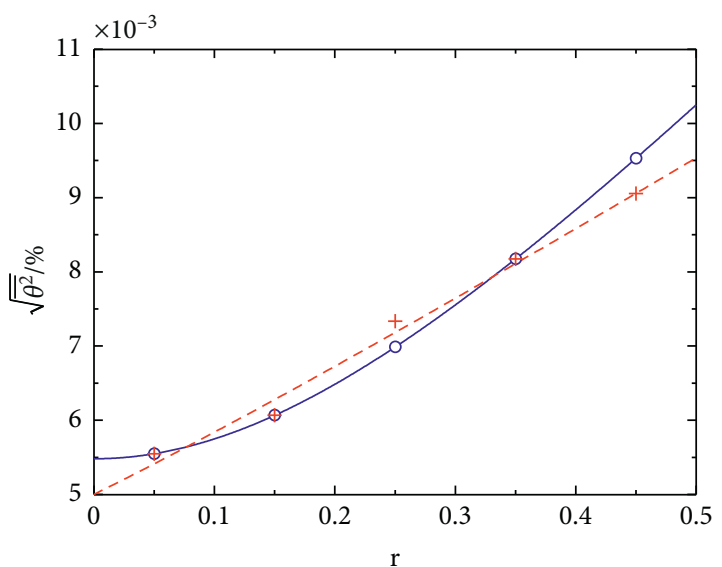

FIgURE 1: Schematic diagram of scatters and fitted curves of ideal and disturbed conditions.

(2) The variances of $m$ and $T_{0}$, their covariance, and correlation coefficient are defined, respectively, as follows:

$$
\begin{aligned}
\sigma_{m}^{2} & =\overline{(\Delta m)^{2}}, \\
\sigma_{T_{0}}^{2} & =\overline{\left(\Delta T_{0}\right)^{2}}, \\
\sigma_{m T_{0}} & =\overline{(\Delta m)\left(\Delta T_{0}\right)} ., \\
\rho_{m T_{0}} & =\frac{\sigma_{m T_{0}}}{\sigma_{m} \sigma_{T_{0}}}, \quad-1 \leq \rho_{m T_{0}} \leq 1 .
\end{aligned}
$$

From equations (18) and (19), the correlation coefficient $\rho_{m T_{0}}$ can be rewritten as

$$
\rho_{m T_{0}}=\frac{\overline{(\Delta m)\left(\Delta T_{0}\right)}}{\sqrt{\overline{(\Delta m)^{2}}} \sqrt{\left(\Delta T_{0}\right)^{2}}}=-\frac{1}{2} \frac{-2 \overline{(\Delta m / \bar{m})\left(\Delta T_{0} / \overline{T_{0}}\right)}}{\sqrt{(\Delta m / \bar{m})^{2}} \sqrt{\left(\Delta T_{0} / \overline{T_{0}}\right)^{2}}} .
$$

Constraints can be established based on the range of the correlation coefficient $\rho_{m T_{0}}$ :

$$
c_{2}^{2}-4 c_{1} c_{3} \leq 0 .
$$

In summary, the problem of hyperbola fitting of twodimensional scattered points can be converted to the following optimization problem:

$$
\begin{aligned}
& \underset{C}{\arg \min } S(\mathbf{C}), \\
& \text { s.t. } \quad c_{1}>0 \& c_{3}>0 \& c_{2}^{2}-4 c_{1} c_{3} \leq 0 .
\end{aligned}
$$

3.2. The LPNN Method. To solve the nonlinear inequality constrained optimization problem in equation (22), the LPNN method is applied [33]. The inequality constraints can be rewritten as follows:

$$
\begin{aligned}
& g_{1}(\mathbf{C})=-c_{1}+\tau \leq 0, \\
& g_{2}(\mathbf{C})=-c_{3}+\tau \leq 0, \\
& g_{3}(\mathbf{C})=c_{2}^{2}-4 c_{1} c_{3} \leq 0,
\end{aligned}
$$

where $\tau$ is the correction value of coefficients $c_{1}$ and $c_{3}$ and $\tau=10^{-10}$. Its value is determined according to the flow quality values of the conventional wind tunnels. The Lagrange function $\mathrm{L}$ with inequality constraints is defined as

$$
L(\mathbf{C}, \boldsymbol{v}, \boldsymbol{\lambda})=S(\mathbf{C})+\sum_{i=1}^{3} \lambda_{i}\left[g_{i}(\mathbf{C})+v_{i}^{2}\right],
$$

where $\lambda=\left[\lambda_{1}, \lambda_{2}, \lambda_{3}\right]^{T}$ are the constraint coefficients and $\lambda_{i} \geq 0$ for $i=1,2,3 . \nu=\left[v_{1}, v_{2}, v_{3}\right]^{T}$ are the relaxation variables. There are three types of neurons in the LPNN method: variable neurons control $\mathbf{C}$, Lagrange neurons $\operatorname{control} \lambda$, and relaxation neurons control $\nu$. According to the KKT condition, the state equations of neurons can be obtained as follows:

$$
\begin{aligned}
\frac{\mathrm{d} \mathbf{C}}{\mathrm{d} t} & =-\frac{\partial L(\mathbf{C}, \boldsymbol{v}, \boldsymbol{\lambda})}{\partial \mathbf{C}}, \\
\frac{\mathrm{d} \boldsymbol{v}}{\mathrm{d} t} & =-\frac{\partial L(\mathbf{C}, \boldsymbol{v}, \boldsymbol{\lambda})}{\partial \boldsymbol{v}}, \\
\frac{\mathrm{d} \boldsymbol{\lambda}}{\mathrm{d} t} & =\frac{\partial L(\mathbf{C}, \boldsymbol{v}, \boldsymbol{\lambda})}{\partial \boldsymbol{\lambda}},
\end{aligned}
$$

where $t$ is characteristic time. The Euler iteration equation is

$$
\begin{aligned}
& \mathbf{C}^{k+1}=\mathbf{C}^{k}-\frac{\partial L\left(\mathbf{C}^{k}, \boldsymbol{v}^{k}, \lambda^{k}\right)}{\partial \mathbf{C}^{k}} \cdot \Delta t, \\
& \boldsymbol{\nu}^{k+1}=\boldsymbol{v}^{k}-\frac{\partial L\left(\mathbf{C}^{k}, \boldsymbol{v}^{k}, \boldsymbol{\lambda}^{k}\right)}{\partial \boldsymbol{v}^{k}} \cdot \Delta t, \\
& \boldsymbol{\lambda}^{k+1}=\boldsymbol{\lambda}^{k}+\frac{\partial L\left(\mathbf{C}^{k}, \boldsymbol{v}^{k}, \boldsymbol{\lambda}^{k}\right)}{\partial \boldsymbol{\lambda}^{k}} \cdot \Delta t,
\end{aligned}
$$

where $k$ is the number of iterations and $\Delta t$ is the iteration time step. The component form of equation (26) is

$$
\begin{aligned}
c_{i}^{k+1} & =c_{i}^{k}-\left[\frac{\partial S\left(\mathbf{C}^{k}\right)}{\partial c_{i}^{k}}+\sum_{j=1}^{3} \lambda_{j}^{k} \frac{\partial g_{j}\left(\mathbf{C}^{k}\right)}{\partial c_{i}^{k}}\right] \cdot \Delta t, \quad i=1,2,3, \\
\nu_{l}^{k+1} & =v_{l}^{k}-2 \lambda_{l}^{k} \nu_{l}^{k} \cdot \Delta t, \quad l=1,2,3, \\
\lambda_{j}^{k+1} & =\lambda_{j}^{k}+\left[g_{j}\left(\mathbf{C}^{k}\right)+\left(\nu_{j}^{k}\right)^{2}\right] \cdot \Delta t, \quad i=1,2,3,
\end{aligned}
$$

The neural network architecture diagram of the entire iteration process is shown in Figure 2.

3.3. Stability Analysis of the LPNN Method. According to the literature [33], when performing stability analysis, it is necessary to verify that the iterative equilibrium point 


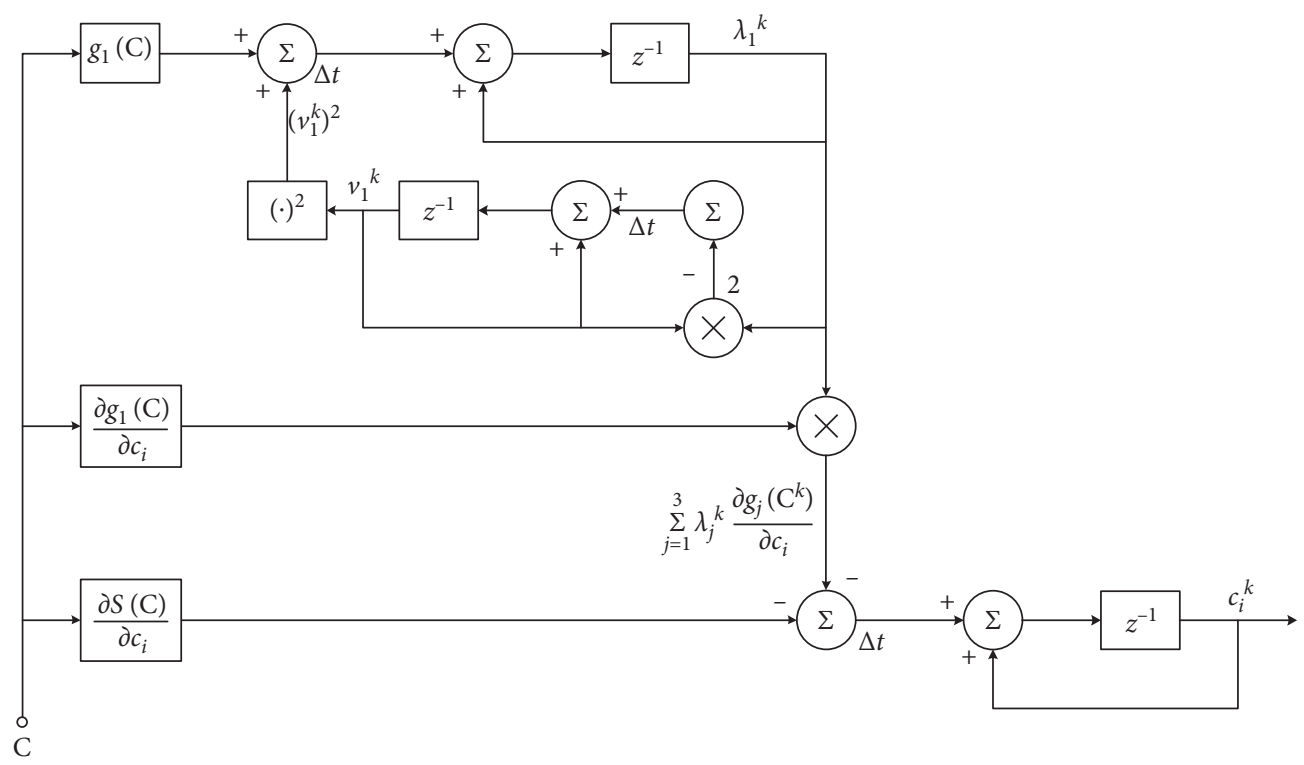

FIGURE 2: Architecture diagram of LPNN for inequality constrained optimization problem.

obtained by the LPNN method is the asymptotically stable point of the neural network. Let the iterative equilibrium point be $\left(\mathbf{C}^{*}, \nu^{*}, \lambda^{*}\right)$, and this point can be obtained by the following equation:

$$
\begin{aligned}
{\left[\begin{array}{l}
\frac{\mathrm{d} \overline{\mathbf{C}}}{\mathrm{d} t} \\
\frac{\mathrm{d} \lambda}{\mathrm{d} t}
\end{array}\right] } & =-\left[\begin{array}{cc}
\nabla_{\overline{\mathrm{CC}}}^{2} L\left(\mathbf{C}^{*}, \boldsymbol{v}^{*}, \boldsymbol{\lambda}^{*}\right) & \nabla_{\overline{\mathbf{C}}} g\left(\mathbf{C}^{*}, \boldsymbol{v}^{*}\right) \\
-\nabla_{\overline{\mathbf{C}}} g\left(\mathbf{C}^{*}, \boldsymbol{v}^{*}\right) & 0
\end{array}\right] \cdot\left[\begin{array}{c}
\overline{\mathbf{C}}-\overline{\mathbf{C}}^{*} \\
\lambda-\lambda^{*}
\end{array}\right] \\
& =-\mathbf{G} \cdot\left[\begin{array}{l}
\overline{\mathbf{C}}-\overline{\mathbf{C}}^{*} \\
\lambda-\lambda^{*}
\end{array}\right],
\end{aligned}
$$

where $\overline{\mathbf{C}}=\left[\mathbf{C}^{T}, v^{T}\right]^{T}$ and $g_{i}(\mathbf{C}, v)=g_{i}(\mathbf{C})+v_{i}^{2}$ for $i=1,2,3$. Additionally, we have

$$
\nabla_{\overline{\mathbf{C}}} g\left(\mathbf{C}^{*}, \boldsymbol{v}^{*}\right)=\left[\begin{array}{l}
\nabla_{\mathbf{C}} g\left(\mathbf{C}^{*}, \boldsymbol{v}^{*}\right) \\
\nabla_{\boldsymbol{v}} g\left(\mathbf{C}^{*}, \boldsymbol{v}^{*}\right)
\end{array}\right]=\left[\begin{array}{ccc}
-1 & 0 & -4 c_{3}^{*} \\
0 & 0 & 2 c_{2}^{*} \\
0 & -1 & -4 c_{1}^{*} \\
2 v_{1}^{*} & 0 & 0 \\
0 & 2 v_{2}^{*} & 0 \\
0 & 0 & 2 v_{3}^{*}
\end{array}\right] .
$$

$\nabla_{\overline{\mathbf{C}}} g\left(\mathbf{C}^{*}, v^{*}\right)$ can be easily verified to be a full column rank. To be more precise, the gradients above at the equilibrium point are linearly independent, which means that $\overline{\mathbf{C}}^{*}$ is a regular point and $\left(\mathbf{C}^{*}, v^{*}, \lambda^{*}\right)$ is a Kuhn-Tucker point of the Lagrange function L [33]; namely, the following equation holds:

$$
\nabla_{\mathrm{CC}}^{2} L\left(\mathbf{C}^{*}, \boldsymbol{v}^{*}, \lambda^{*}\right)>0
$$

The specific form of $\nabla \frac{2}{\mathrm{CC}} L\left(\mathbf{C}^{*}, \nu^{*}, \lambda^{*}\right)$ in matrix $G$ in equation (28) is

$$
\nabla_{\overline{\mathrm{CC}}}^{2} L\left(\mathbf{C}^{*}, \boldsymbol{v}^{*}, \boldsymbol{\lambda}^{*}\right)=\left[\begin{array}{cccc}
\nabla_{\mathrm{CC}}^{2} L\left(\mathbf{C}^{*}, \boldsymbol{v}^{*}, \lambda^{*}\right) & 0 & 0 & 0 \\
0 & 2 \lambda_{1}^{*} & 0 & 0 \\
0 & 0 & 2 \lambda_{2}^{*} & 0 \\
0 & 0 & 0 & 2 \lambda_{3}^{*}
\end{array}\right] .
$$

According to the results of equation (30) and $\lambda_{i} \geq 0$ for $i=1,2,3$, we can say that $\nabla_{\overline{\mathrm{CC}}}^{2} L\left(\mathbf{C}^{*}, \nu^{*}, \lambda^{*}\right)$ is a strict positive definite matrix and the coefficient matrix $\mathbf{G}$ in equation (28) is a negative semidefinite matrix, which means that the equilibrium point $\left(\mathbf{C}^{*}, \nu^{*}, \lambda^{*}\right)$ is the asymptotically stable point of the network.

\section{Results and Analysis}

4.1. Results of the Proposed Algorithm. Three sets of typical turbulence level measurement test results are selected to be processed. The independent variable and dependent variable data in equation (3) are shown in Table 1.

The LPNN method is used to iteratively solve the turbulence level corresponding to the three sets of test data above in Table 1. Considering convergence speed, the initial value of iteration is selected as $10^{-4}, 10^{-5}, 10^{-6}$, and $10^{-7}$, respectively, and the iteration time step is $\Delta t=10^{-8}$. The iteration results of variable $\mathbf{C}$ are shown in Figure 3. The iteration results of variables $v$ and $\lambda$ have no concern with solving the turbulence level, so they are not listed here.

From Figure 3, we can easily find that variable $\mathbf{C}$ converges to a fixed value within approximate $4 \times 10^{6}$ steps, and consistent results can be obtained with different initial values. The results indicate good and stable convergence of the LPNN method. 
TABLE 1: Data on turbulence level measurement test.

\begin{tabular}{|c|c|c|c|c|c|}
\hline \multicolumn{2}{|l|}{$M=0.449$} & \multicolumn{2}{|c|}{$M=0.516$} & \multicolumn{2}{|c|}{$M=0.599$} \\
\hline$R$ & $\sqrt{\overline{\theta^{2}}} / \%$ & $r$ & $\sqrt{\overline{\theta^{2}}} / \%$ & $r$ & $\sqrt{\overline{\theta^{2}}} / \%$ \\
\hline 0.146 & 0.093 & 0.147 & 0.058 & 0.146 & 0.074 \\
\hline 0.177 & 0.110 & 0.179 & 0.068 & 0.178 & 0.073 \\
\hline 0.214 & 0.112 & 0.217 & 0.080 & 0.215 & 0.079 \\
\hline 0.257 & 0.111 & 0.261 & 0.092 & 0.259 & 0.100 \\
\hline 0.413 & 0.181 & 0.426 & 0.159 & 0.421 & 0.180 \\
\hline 0.742 & 0.314 & 0.788 & 0.273 & 0.773 & 0.279 \\
\hline
\end{tabular}

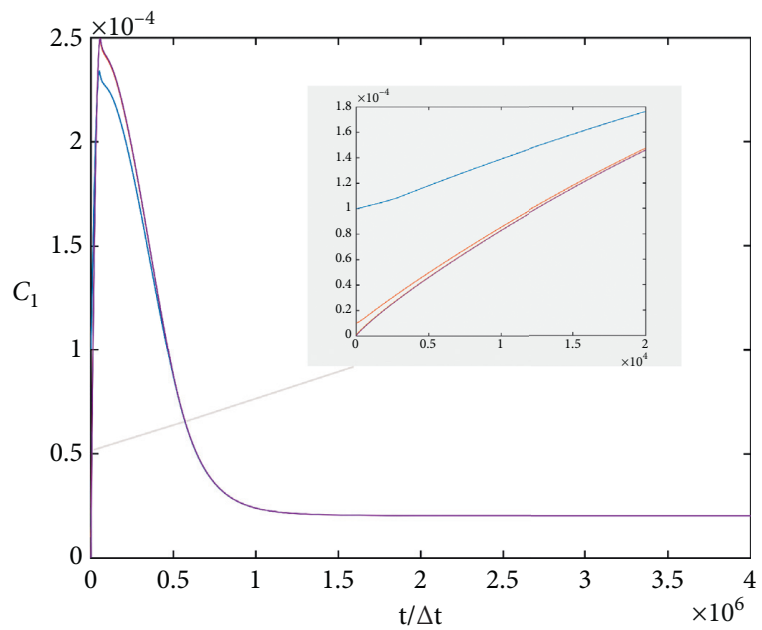

(a)

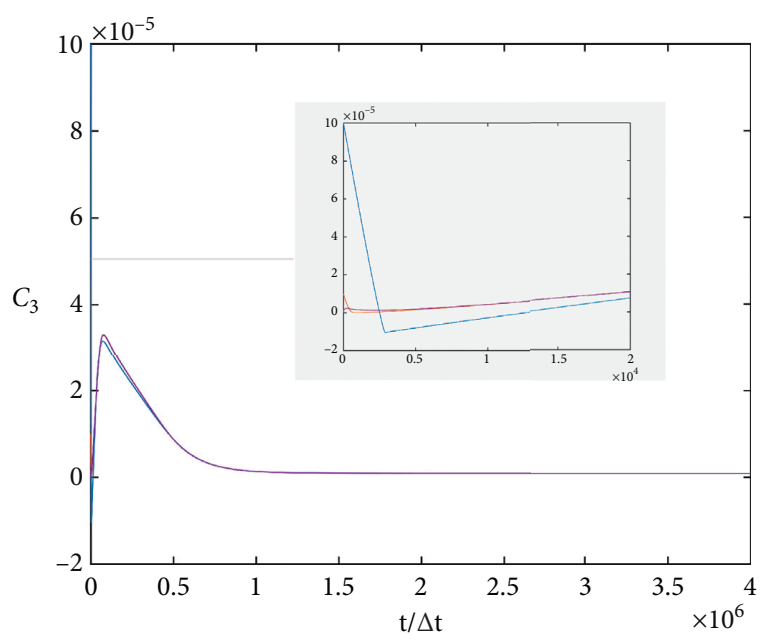

(c)

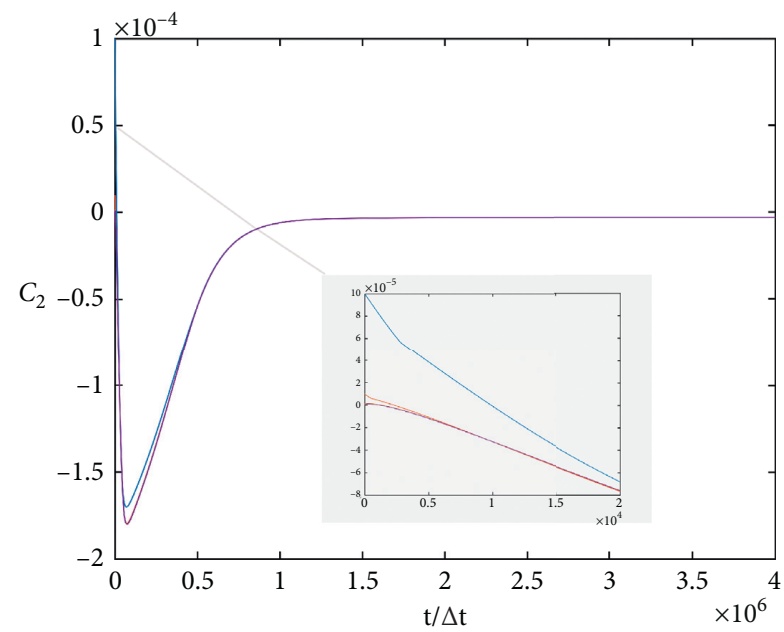

(b)

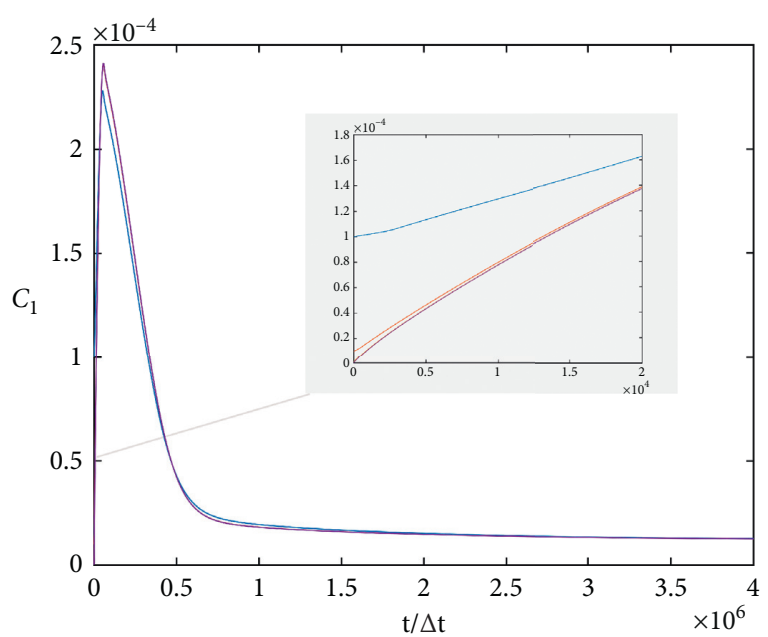

(d)

FIgURE 3: Continued. 


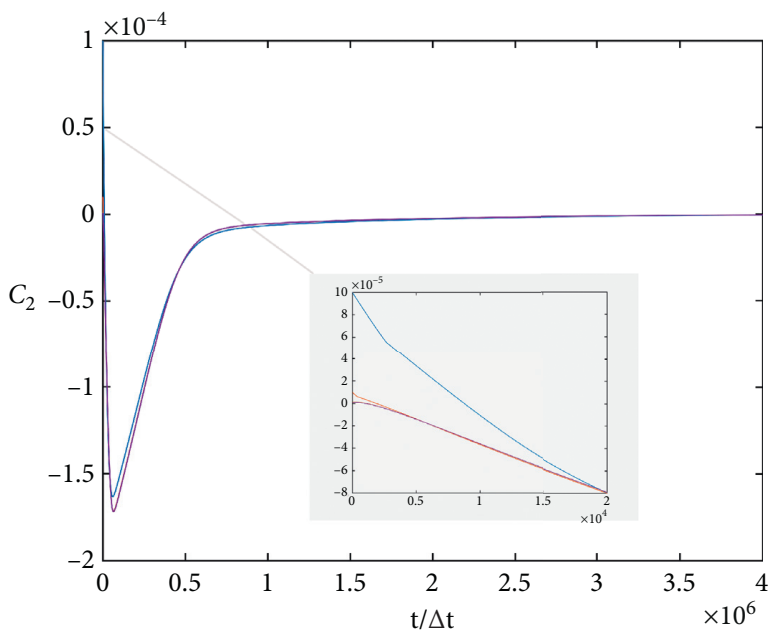

(e)

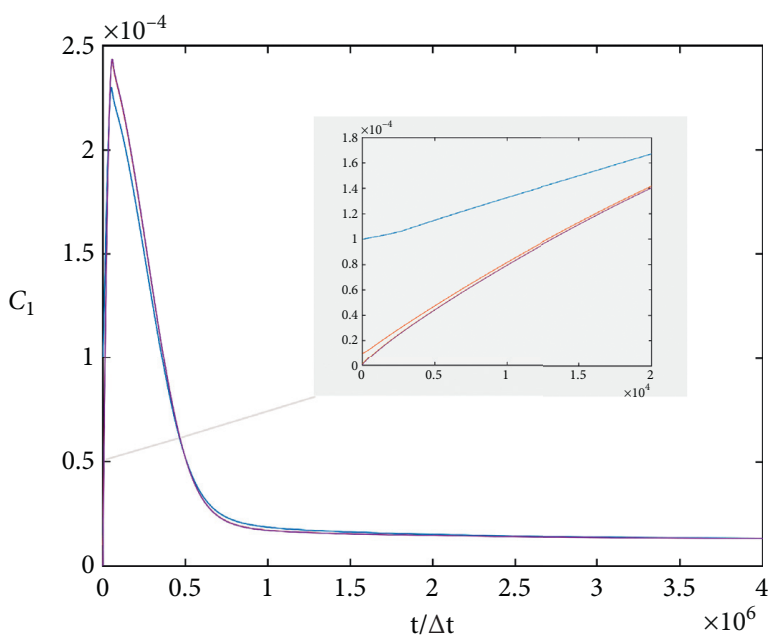

(g)

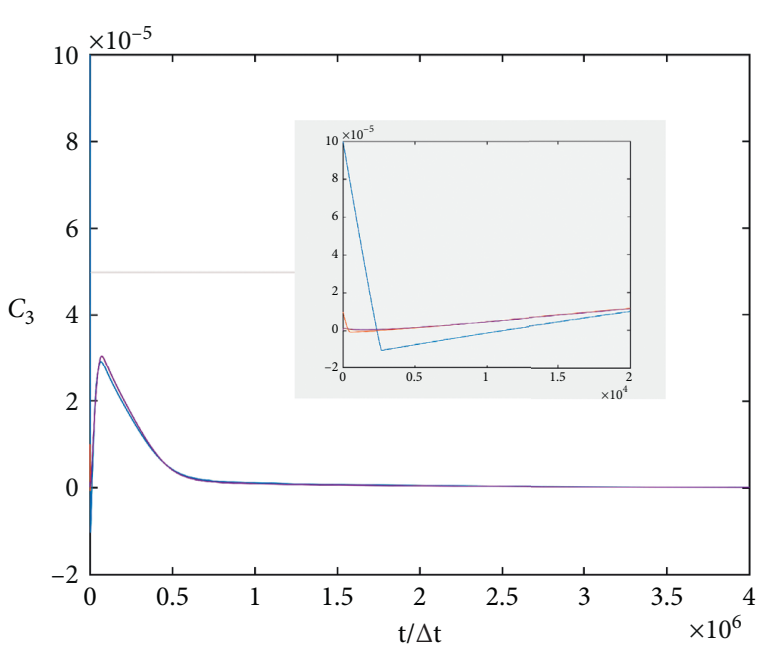

(f)

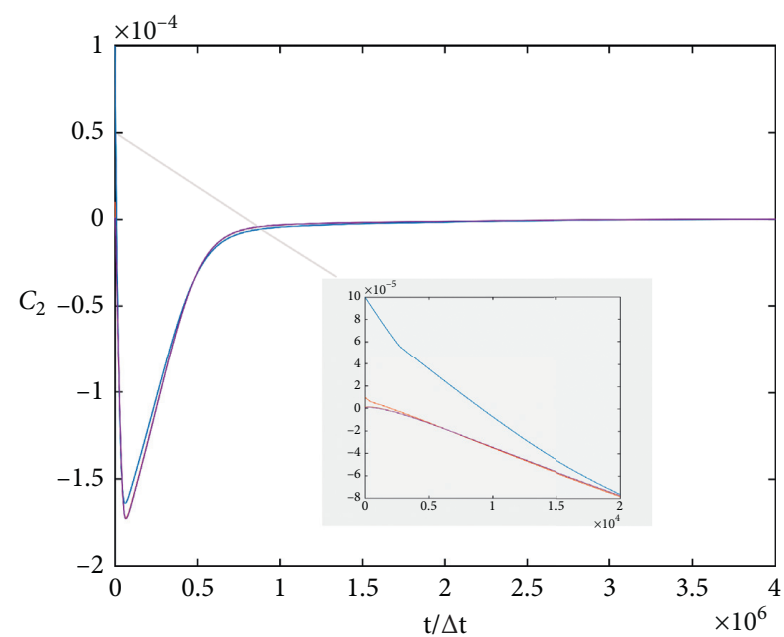

(h)

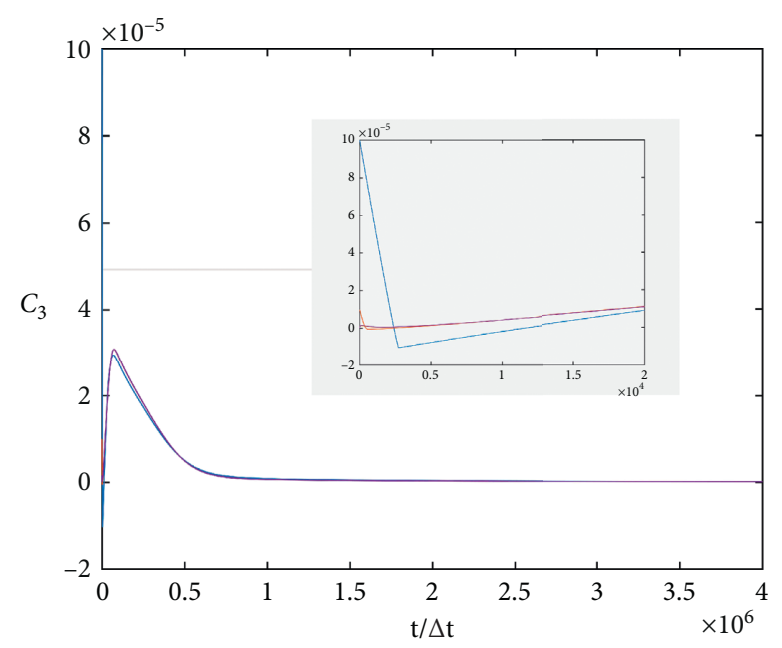

(i)

Figure 3: Results of variables from the iteration process. (a) $C_{1}$ for $M=0.449$. (b) $C_{2}$ for $M=0.449$. (c) $C_{3}$ for $M=0.449$. (d) $C_{1}$ for $M=0.516$. (e) $C_{2}$ for $M=0.516$. (f) $C_{3}$ for $M=0.516$. (g) $C_{1}$ for $M=0.599$. (h) $C_{2}$ for $M=0.599$. (i) $C_{3}$ for $M=0.599$. 


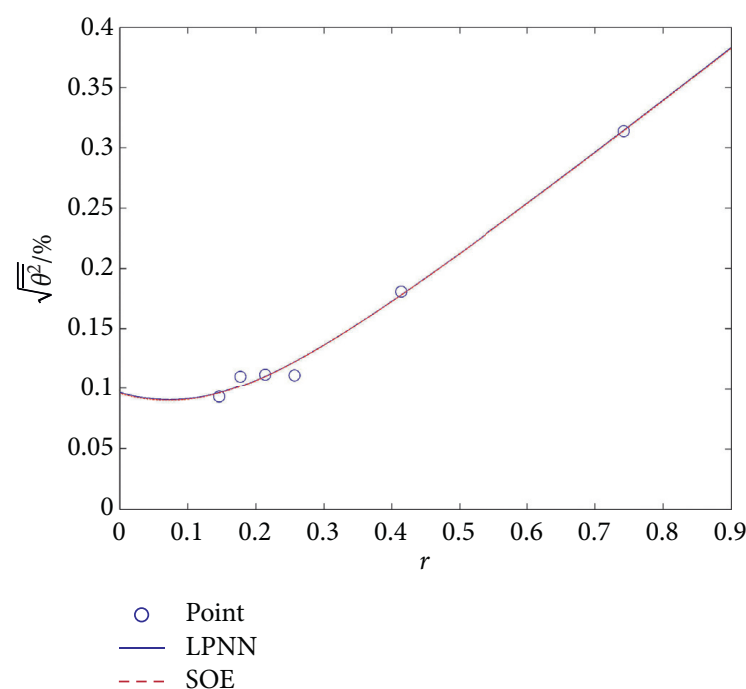

(a)

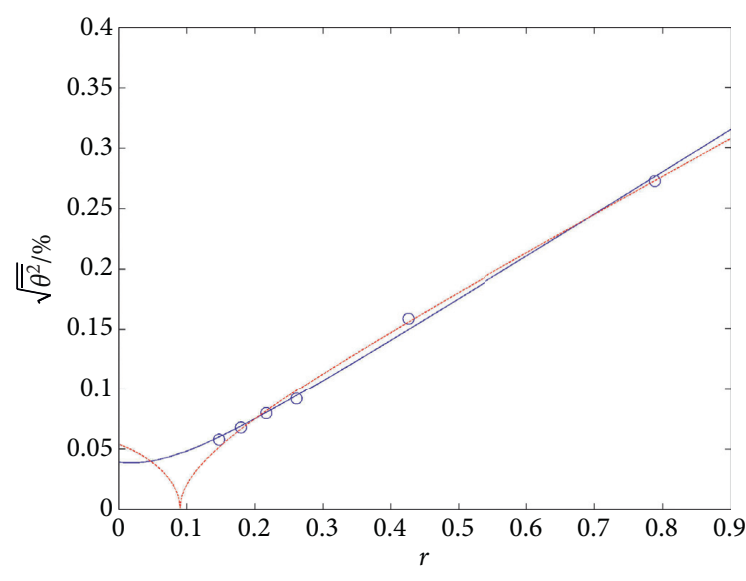

○ Point

$\begin{array}{ll}- & \text { LPNN } \\ -- & \text { SOE }\end{array}$

(b)

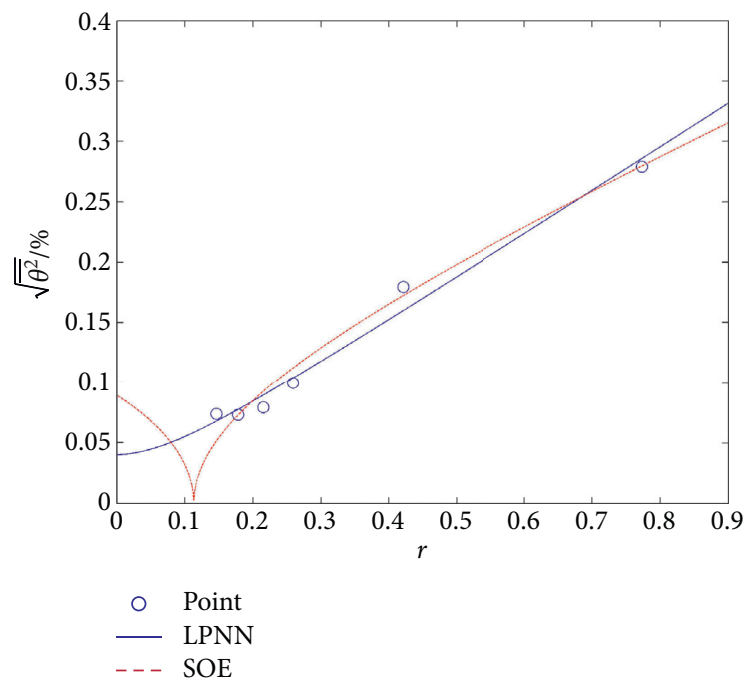

(c)

FIgure 4: Comparison of hyperbola fitting from LPNN and SOE results. (a) $M=0.449$. (b) $M=0.516$. (c) $M=0.599$.

4.2. Comparison with the SOE Method. The comparison results of the fitted hyperbola obtained by the LPNN method and the traditional SOE method are shown in Figure 4. The turbulence level and other flow qualities by the two methods are shown in Table 2.

For the case $M=0.449$, Figure 4 (a) shows that the scattered points of turbulence level measurement test results basically conform to the hyperbolic distribution, and the fitted hyperbolae obtained by the LPNN method and the traditional SOE method basically coincide. The results in Table 2 show that the turbulence level and other flow qualities are basically equal for $M=0.449$. Therefore, in the case when the scattered points conform to the hyperbolic distribution, both the LPNN method and the SOE method can be used to solve the turbulence level accurately.
For the cases $M=0.516$ and $M=0.599$, Figures $4(\mathrm{~b})$ and 4 (c) show that the scattered points deviate from the hyperbolic distribution due to the unsteadiness of the flow, and there are even straight lines and other conic curves for the scattered points distribution. The fitting curve obtained by the traditional SOE method is an ellipse, which is inconsistent with the theoretical results from equation (3). Table 2 shows the high goodness of fitting values and negative $C_{3}$ values for the SOE results of $M=0.516$ and $M=0.599 . C_{3}$ represents the mean square value of $T_{0}$; thus, $C_{3}$ should be positive for all situations according to its physical meaning. However, the results of $C_{3}$ obtained by the SOE method are negative, which indicate that overfitting has occurred for $M=0.516$ and $M=0.599$. The scattered points are overfitted into ellipses by the SOE method, which results in negative $C_{3}$ 
TABLE 2: Comparison of flow quality from LPNN and SOE results.

\begin{tabular}{|c|c|c|c|c|c|c|}
\hline & Method & $(\Delta m / \bar{m})^{2}\left(C_{1}\right)$ & $-2(\Delta m / \bar{m})\left(\Delta T_{0} / \overline{T_{0}}\right)\left(C_{2}\right)$ & $\left(\Delta T_{0} / \overline{T_{0}}\right)^{2}\left(C_{3}\right)$ & $T u(\%)$ & Goodness of fitting \\
\hline \multirow{2}{*}{$M=0.449$} & LPNN & $2.03 \times 10^{-5}$ & $-2.97 \times 10^{-6}$ & $9.27 \times 10^{-7}$ & 0.54 & 0.9981 \\
\hline & SOE & $2.02 \times 10^{-5}$ & $-2.90 \times 10^{-6}$ & $9.11 \times 10^{-7}$ & 0.54 & 0.9981 \\
\hline \multirow{2}{*}{$M=0.516$} & LPNN & $1.26 \times 10^{-5}$ & $-4.51 \times 10^{-7}$ & $1.54 \times 10^{-7}$ & 0.44 & 0.9974 \\
\hline & SOE & $9.40 \times 10^{-6}$ & $2.41 \times 10^{-6}$ & $-2.93 \times 10^{-7}$ & 0.46 & 0.9992 \\
\hline \multirow{2}{*}{$M=0.599$} & LPNN & $1.33 \times 10^{-5}$ & $9.36 \times 10^{-8}$ & $1.61 \times 10^{-7}$ & 0.48 & 0.9864 \\
\hline & SOE & $6.28 \times 10^{-6}$ & $6.29 \times 10^{-6}$ & $-7.92 \times 10^{-7}$ & 0.52 & 0.9936 \\
\hline
\end{tabular}

values. In this situation, although equations (12) and (13) can still be used to solve turbulence levels, the results are not true values.

The fitting curve for the cases $M=0.516$ and $M=0.599$ obtained by the LPNN method is still hyperbola with the influence of the unsteadiness of the flow, and the goodness of fitting values are high, which is consistent with the theoretical results. From the results, we can easily find that the LPNN method is better than the SOE method, and the results verify the feasibility of applying the proposed algorithm to solve the turbulence level in wind tunnels.

\section{Conclusions}

In this paper, we propose a new hyperbola fitting algorithm for turbulence level measurement test data, based on the Lagrange programming neural network method, to solve the turbulence level in wind tunnels iteratively. The LPNN method is stable and the equilibrium point is proved to be asymptotically stable. The results show that, better than the SOE method, the LPNN method can be utilized to solve turbulence level, and the results will not be affected by the unsteadiness of the flow. The results of this paper will be mainly used to solve the turbulence level more precisely to ensure the high accuracy of wind tunnel test results.

Future research will continue to further develop the hyperbola fitting algorithm on the basis of the LPNN method to improve the accuracy of turbulence level results. In addition, the LPNN method will also be improved to be applied in data processing for wind tunnel test results.

\section{Data Availability}

The data used to support the findings of this study are included within the tables of this article. More turbulence level measurement test data are available from the corresponding author upon request.

\section{Conflicts of Interest}

The authors declare that there are no conflicts of interest regarding the publication of this paper.

\section{References}

[1] W. D. Harvey, P. C. Stainback, and F. K. Owen, Evaluation of Flow Quality in Two Large Nasa Wind Tunnels at Transonic Speeds: NASA-TP-1737, NASA, Hampton, Virginia, 1980.
[2] B. Rasuo, "The influence of Reynolds and Mach numbers on two-dimensional wind-tunnel testing: an experience," The Aeronautical Journal, vol. 115, no. 1166, pp. 249-254, 2011.

[3] B. Rasuo, "Scaling between wind tunnels-results accuracy in two-dimensional testing," Transactions of the Japan Society for Aeronautical and Space Sciences, vol. 55, no. 2, pp. 109-115, 2012.

[4] D. Damljanovic, J. Isakovic, and B. Rasuo, "T-38 wind-tunnel data quality assurance based on testing of a standard model," Journal of Aircraft, vol. 50, no. 4, pp. 1141-1149, 2013.

[5] D. Damljanovic, B. Rasuo, D. Vukovic et al., "Hypervelocity ballistic reference models as experimental supersonic test cases," Aerospace Science and Technology, vol. 52, no. 5, pp. 189-197, 2016.

[6] D. Damljanovic, D. Vukovic, G. Ocokoljic et al., "A study of wall-interference effects in wind-tunnel testing of a standard model at transonic speeds," in Proceedings of the 30th Congress of the International Council of the Aeronautical Sciences, Daejeon, Korea, September 2016.

[7] G. Ocokoljic, B. Rasuo, and M. Kozic, "Supporting system interference on aerodynamic characteristics of an aircraft model in a low-speed wind tunnel," Aerospace Science and Technology, vol. 64, pp. 133-146, 2017.

[8] F. K. Owen, T. K. McDevitt, and D. G. Morgan, Wind Tunnel Angle of Attack Measurements Using an Optical Model Attitude System: AIAA-2000-0414, AIAA, Reno, Nevada, 2000.

[9] F. K. Owen and A. K. Owen, Effects of Freestream Flow Quality on Boundary Layer Transition in the National Transonic Facility: AIAA-2013-1135, AIAA, Grapevine, TX, USA, 2013.

[10] S. L. Treon, F. W. Steinle, and W. R. Hofstetter, Data Correlation from Investigations of a High-Subsonic Speed Transport Aircraft Model in Three Major Transonic Wind Tunnels: AIAA-69-794, AIAA, Los Angeles, CA, USA, 1969.

[11] H. L. Dryden, G. B. Schubauer, and W. C. Mock, Measurements of Intensity and Scale of Wind-Tunnel Turbulence and their Relation to the Critical Reynolds Number of Spheres: Naca-Report-581, National Advisory Committee for Aeronautics, Washington, DC, USA, 1937.

[12] V. Zinoviev and V. Lebiga, Application of Hot-Wire Technology in a Blowdown Type Transonic Wind Tunnel: AIAA2001-0308, AIAA, Reno, Nevada, 2001.

[13] H. Quix, J. Quest, and C. Brzek, Hot-Wire Measurements in Cryogenic Environment: AIAA-2011-880, AIAA, Orlando, FL, USA, 2011.

[14] D. Masutti, E. Spinosa, O. Chazot, and M. Carbonaro, "Disturbance level characterization of a hypersonic blowdown facility,” AIAA Journal, vol. 50, no. 12, pp. 2720-2730, 2012.

[15] Y. F. Du, J. Lin, and H. S. Ma, "Measurement technique for turbulence level in compressible fluid by changing overheat ratio of hot-wire anemometer," Acta Aeronautica et Astronautica Sinica, vol. 38, no. 11, p. 121236, 2017. 
[16] R. O. Duda and P. E. Hart, "Use of the Hough transformation to detect lines and curves in pictures," Communications of the $A C M$, vol. 15, no. 1, pp. 11-15, 1972.

[17] D. H. Ballard, "Generalizing the Hough transform to detect arbitrary shapes," Pattern Recognition, vol. 13, no. 2, pp. 111-122, 1981.

[18] J. Princen, J. Illingworth, and J. Kittler, "A formal definition of the Hough transform: properties and relationships," Journal of Mathematical Imaging and Vision, vol. 1, no. 2, pp. 153-168, 1992.

[19] G. Borgioli, L. Capineri, P. Falorni, S. Matucci, and C. G. Windsor, "The detection of buried pipes from time-offlight radar data," IEEE Transactions on Geoscience and Remote Sensing, vol. 46, no. 8, pp. 2254-2266, 2008.

[20] Q. M. Zhao, W. Li, and H. L. Zhou, "Hyperbola fitting and dimension inversion of cylindrical target based on modified Hough model," Journal of PLA University of Science and Technology (Natural Science Edition), vol. 13, no. 4, pp. 365370, 2012.

[21] P. D. Sampson, "Fitting conic sections to "very scattered" data: an iterative refinement of the bookstein algorithm," Computer Graphics and Image Processing, vol. 18, no. 1, pp. 97-108, 1982.

[22] R. Safaee-Rad, I. Tchoukanov, B. Benhabib, and K. C. Smith, "Accurate parameter estimation of quadratic curves from grey-level images," CVGIP: Image Understanding, vol. 54, no. 2, pp. 259-274, 1991.

[23] K. Kanatani, "Statistical bias of conic fitting and renormalization," IEEE Transactions on Pattern Analysis and Machine Intelligence, vol. 16, no. 3, pp. 320-326, 1994.

[24] S. J. Ahn, W. Rauh, and H.-J. Warnecke, "Least-squares orthogonal distances fitting of circle, sphere, ellipse, hyperbola, and parabola," Pattern Recognition, vol. 34, no. 12, pp. 2283-2303, 2001.

[25] X. Tu, X. Lei, W. Ma, X. Zuo, and R. Man, "Hyperbola minimum-zone evaluation and fitting based on geometryoptimised search algorithm," Measurement, vol. 127, pp. 205-209, 2018.

[26] V. A. Lebiga, V. N. Zinov'ev, and A. Y. Pak, "Using a hot-wire anemometer for measurement of characteristics of a random acoustic field in compressible flows," Journal of Applied Mechanics and Technical Physics, vol. 43, no. 3, pp. 488-492, 2002.

[27] V. N. Zinovev, V. A. Lebiga, and A. Y. Pak, "Preliminary results of flow fluctuation measurements in the cryogenic transonic wind tunnel," Progress in Flight Physics, vol. 3, pp. 3-14, 2012.

[28] S. R. C. Ali, J. Wu, and R. Radespiel, High-frequency Measurements of Acoustic and Entropy Disturbances in a Hypersonic Wind Tunnel: AIAA-2014-2644, AIAA, Atlanta, GA, USA, 2014.

[29] J. Wu, P. Zamre, and R. Radespiel, "Flow quality experiment in a tandem nozzle wint tunnel at Mach 3," Experiments in Fluids, vol. 56, p. 20, 2015.

[30] T. Schilden, W. Schroder, and S. R. C. Ali, "Analysis of acoustic and entropy disturbances in a hypersonic wind tunnel," Physics of Fluids, vol. 28, p. 56104, 2016.

[31] F. Munoz, J. Wu, and R. Radespiel, Freestream Disturbances Characterization in Ludwieg Tubes at Mach 6, AIAA SciTech Forum, San Diego, CA, USA, 2019.

[32] Y. F. Du, J. Lin, and X. N. Wang, "Measurement technique optimization of turbulence level in compressible fluid by changing overheat ratio of hot wire anemometer," Acta Aeronautica et Astronautica Sinica, vol. 40, no. 12, Article ID 123067, 2019.
[33] S. Zhang and A. G. Constantinides, "Lagrange programming neural networks," IEEE Transactions on Circuits and Systems II: Analog and Digital Signal Processing, vol. 39, no. 7, pp. 441-452, 1992. 